\title{
An Uplink Bandwidth Management Framework for IEEE 802.16 with QoS Guarantees
}

\author{
Mohamad El Masri, Slim Abdellatif, and Guy Juanole \\ CNRS, LAAS, Université de Toulouse, 7, Avenue du Colonel Roche, F-31077, \\ Toulouse, France \\ Université de Toulouse, UPS, INSA, INP, LAAS, F-31077 Toulouse, France \\ \{masri,slim, juanole\}@laas.fr
}

\begin{abstract}
This paper presents a novel uplink bandwidth management framework for IEEE 802.16 WiMAX. This framework is destined to be used in a scenario where a large number of connections are simultaneously active, as may be the case when WiMAX is used as a backhaul for WiFi hotspots. In such a scenario we propose a simple and efficient scheduling discipline for the WiMAX uplink traffic. We also propose an admission control algorithm and a modification of the complex bandwidth request mechanisms. The framework we propose in this paper was analyzed: simulation results show a significant performance improvement in terms of overall throughput and delay when compared to recently published work.
\end{abstract}

Keywords: WiMAX, Quality of Service, Bandwidth Management, Scheduling.

\section{Introduction}

The IEEE 802.16 standard [1] (on which is based the Worldwide Interoperability for Microwave Access - WiMAX) defines the physical layer (PHY) and Medium Access Control (MAC) layer for Broadband Wireless Point to Multipoint and Mesh Access. WiMAX is seen by many as the solution for broadband wireless access requiring Quality of Service. A point-to-multipoint (PMP) WiMAX network is structured in a centralized Base Station (BS) and several Subscriber Stations (SS). The BS's role is to manage its Downlink access (DL) and the different SSs Uplink access (UL). Access to the medium for uplink data transfer is done in a connection oriented contention-less polling fashion. In early WiMAX forum documents such as [2], a suggested business case scenario was the use of the IEEE 802.16 access as a WiFi Hot Spot Backhaul. In such a scenario, a scalability issue should be analyzed. The lack of scalability will induce a downgrade in the Quality of Service offered to the different time sensitive flows using the network. It is clear that in such a scenario, leaving to the WiMAX BS the responsibility of managing the bandwidth provision of each connection individually becomes a serious scalability issue. This is also the case in other scenarios presented in the business case analysis 2]: for example serving high speed Internet access in rural areas where DSL services are not available.

L. Fratta et al. (Eds.): NETWORKING 2009, LNCS 5550, pp. 651663, 2009.

(C) IFIP International Federation for Information Processing 2009 
Several work proposed scheduling algorithms for WiMAX [345]. Others proposed QoS architectures [6] giving scheduling and admission algorithms. But few concentrated on the scalability issue, which is essential for WiMAX to be used in the scenarios we discussed above.

We propose in this paper an uplink bandwidth management framework composed of scheduling algorithms for both the SS and the BS and an admission control algorithm. These algorithms are coupled to a modification of the complex bandwidth request mechanisms of the WiMAX standard we discussed in an earlier paper 7 . The aim of the proposed framework is to have a simpler and more flexible bandwidth management in order to solve the scalability issue while still insuring guarantees on both rate and access delay metrics for time sensitive flows. The paper is organized as follows: we first present different aspects of the WiMAX standard that are of interest for a good understanding of our work and an overview of the related work in the literature. The second section details our framework. The third section presents an analysis of the proposed framework. The paper is then concluded and the perspectives of this work are given.

\section{Bandwidth Provision in WiMAX}

WiMAX access is frame based. It manages separately an Uplink and a Downlink subframe. The subframes are duplexed either in time (TDD) or in frequency (FDD). The BS will send, at the beginning of each downlink subframe, two messages managing the scheduling. The UL-MAP will specify the multiplexing among the SSs (by TDMA or OFDMA) of the uplink subframe. The DL-MAP will specify how the downlink subframe will be organized.

\subsection{Detailing the Services}

WiMAX specifies four scheduling services to which the uplink connections are mapped. An uplink connection, depending on the service it is mapped to, is bound to use a set of rules specifying the way it requests bandwidth and will be served accordingly. The services are: The Unsolicited Grant Service (UGS), The Real-Time Polling Service (rtPS), the non-Real-Time Polling Service (nrtPS) and the Best Effort service (BE). We detail in the following paragraph the request-grant policies that each of the services can use.

\subsection{Bandwidth Requests: The WiMAX Way}

Bandwidth requests are done in WiMAX on a per connection basis. Several ways are available to allow a connection to request bandwidth or to specify needs. Upon establishing a connection, a specification of the flow using the connection is communicated to the BS. This specification can be considered as the initial bandwidth request made by the connection. Other methods to request bandwidth (or to specify the need to be polled) while the connection is active are: 
- a unicast request opportunity is a period of airtime where only the destined connection can express its needs,

- a contention request opportunity is a period where several connections may express their needs in a CSMA/CA fashion contention based access, this kind of opportunities are programmed by the BS if, due to lack of space, it can not program enough unicast request opportunities,

- piggyback requests can be included by some connections in a specific type of headers: the Grant Management subheader,

- this same subheader contains the Poll Me bit which, if set, specifies the need of the SS to be polled for a bandwidth request by the BS.

$U G S$ : A UGS connection is periodically granted air time without having to specifically request it. The amount of the grant is fixed upon set up of the connection, based on the Maximum Sustained traffic of the flow. A UGS connection is not allowed to use any contention based request period and is not allocated unicast request opportunities. If a UGS connection's transmit depth queue is exceeded (due to a lost UL-MAP or due to clock mismatch) it sets in outbound packets the SI bit (Slip Indicator bit) informing the BS of the situation. The PM bit (Poll Me bit) in outbound UGS packets can be used to request polls for other non-UGS connections.

rtPS : An rtPS connection is provided with periodic unicast request opportunities. Those opportunities will be used by the connection to express its needs depending on its queue situation. An rtPS connection is not allowed to use contention request opportunities.

nrtPS : An nrtPS connection is provided with regular unicast request opportunities (the standard specifies an interval on the order of one second or less). An nrtPS connection can also use contention request opportunities.

$B E$ : A BE connection may be granted unicast request opportunities by the BS. It may also use contention request opportunities in order to express its needs.

\subsection{The Grants}

An SS's medium access for Uplink data transmission is done in a contentionless, polling based fashion. Within the BS, a scheduling algorithm, which is not specified in the standard, will build the UL-MAP (map of the transmission opportunities granted for the uplink direction). The UL-MAP is built based on the requests the BS received and on the initial per connection information it possesses. Two grant modes were initially defined by the 802.16 workgroup: GPC (Grant Per Connection) and GPSS (Grant Per Subscriber Station). In GPC mode, the UL-MAP specifies the time range each connection in each SS should individually use. This mode is obsolete. In GPSS mode, transmission opportunity is granted to the SS; an uplink scheduler within the SS will grant each of its connections a range in the granted time. 


\subsection{Overview of the Literature}

The IEEE 802.16 standard does not give any specifications of the different QoS mechanisms to use. Recent work concentrated on these aspects. Architectures focusing on the different uplink scheduling algorithms to be used for the different classes of service were given in 345. A QoS architecture was defined in 6] which instantiated the different blocs of a QoS architecture (admission control, classifiers, schedulers, traffic shaper and different queuing mechanisms). However few concentrated on the scalability issues. The first step in this direction was putting away from the standard the GPC mechanism which was a serious flaw in terms of scalability. Other work [8] concentrated on the optimal duration of the contention period in order to reduce collision probabilities. Our work focuses on the bandwidth management mechanisms. We propose a redesign of the request mechanisms based on an aggregated management. We also provide flexible and simplified scheduling procedures. Our design will offer rate guarantees and latency bounds to sensitive flows by adopting a Latency Rate 9] server behavior.

\section{A Novel Bandwidth Management Framework}

\subsection{Overview of the Framework}

We propose a novel bandwidth management framework for IEEE 802.16 uplink communication. The framework we propose is based on the aggregation of the bandwidth management. The aggregation here concerns the bandwidth management at the BS: instead of having to perform the scheduling on a per connection basis (which would be problematic in our case where a high number of connections will be simultaneously active), we simplify the scheduling at the BS by decentralizing this feature to the SS level, the BS will have to perform the scheduling on a per SS basis. We think that aggregating the bandwidth management will allow us to have a far simpler, more flexible bandwidth management. Our proposal will further be able to give guarantees on the rate and on the access delay of time sensitive flows. The aggregation will also cover the bandwidth request mechanisms. It will use the GPSS grant mode as specified by WiMAX. This is coupled to scheduling algorithms both on the SS side and on the BS side and an admission control algorithm allowing the guarantees to be given. The framework will act towards the flows differently whether they are time sensitive (UGS and rtPS flows), throughput requiring (nrtPS flows), or best effort flows.

The framework works as follows: all uplink flows are subject to admission control at the BS, the sum of the requested rates of all time sensitive flows of an SS (those using UGS or rtPS types of service) defines the contract of the said SS. Instead of having each connection request its own needs in terms of bandwidth, an aggregated request, covering all the needs of an SS is sent out to the BS periodically. An important feature of our proposal is to have the BS answer positively and immediately the amount of requested bandwidth falling within the SS's contract, the grant should come in the frame following the one bearing the request. This is done in order to insure the guarantees on 
rate and delay that the framework offers. The admission control algorithm along with a resource reservation process will be responsible of guaranteeing the whole framework will work correctly. We assume the presence of policing and shaping mechanisms that we will not detail herein. We detail in the following paragraphs the different aspects of the proposed framework.

\subsection{Admission Control Algorithm}

Overview. One feature of our proposal is the guarantees on delays and rate it can give to time sensitive flows. It is thus necessary to have an efficient admission control algorithm working at the BS. The admission control algorithm should guarantee that any time sensitive request falling within an SS's contract will be granted in the frame following the one that carried the request (the contract is defined as the sum of the mean rates of all admitted UGS and rtPS flows). Each new flow of an SS will be individually submitted to admission control (algorithm 1). We separate in the algorithm the procedure of admission of time sensitive flows from the admission procedure of nrtPS flows and Best Effort flows:

- UGS and rtPS flows are to be considered, for the admission control algorithm, individually and with a Frame Size granularity: i.e. the admission control algorithm will reserve for each admitted flow of these types, within all the uplink subframes covering the time of activity of the flow, enough slots (as calculated algo. 1 line 2) to serve the said flow. This is necessary if guarantees on the delay are to be given to UGS and rtPS flows. It should be noted that, since the uplink allocation unit in WiMAX is physical layer dependent, the framework should be adapted to the physical layer used. We call slot the generic uplink allocation unit. This can be a WiMAX minislot for a single carrier physical implementation or a combination of symbols and subchannels for OFDM and OFDMA based physical implementations.

- The cost in terms of slots of an nrtPS flow requesting admission will not be considered individually but rather as a fraction of all nrtPS flows in the network (see algo. 1 line 10-11). nrtPS flows are in no need for delay guarantees; they are to be served with the rate they ask for, however no constraint on the delay is to be enforced. Thus, the slot reservation for nrtPS flows is done on a larger scale than that of time sensitive flows.

- Best Effort flows will always be accepted (see algo. 1 line 19-20): since no guarantees are to be given to such flows and since the medium access in WiMAX is controlled and contention-less, new Best Effort flows will not affect other flows' performance. In order to avoid BE flows starvation, a fixed percentage of uplink bandwidth will be reserved (i.e. the admission control will only consider the remaining bandwidth when admitting other types of connections).

The Algorithm. The admission control algorithm described in 1 will need a number of parameters that we define herein: 


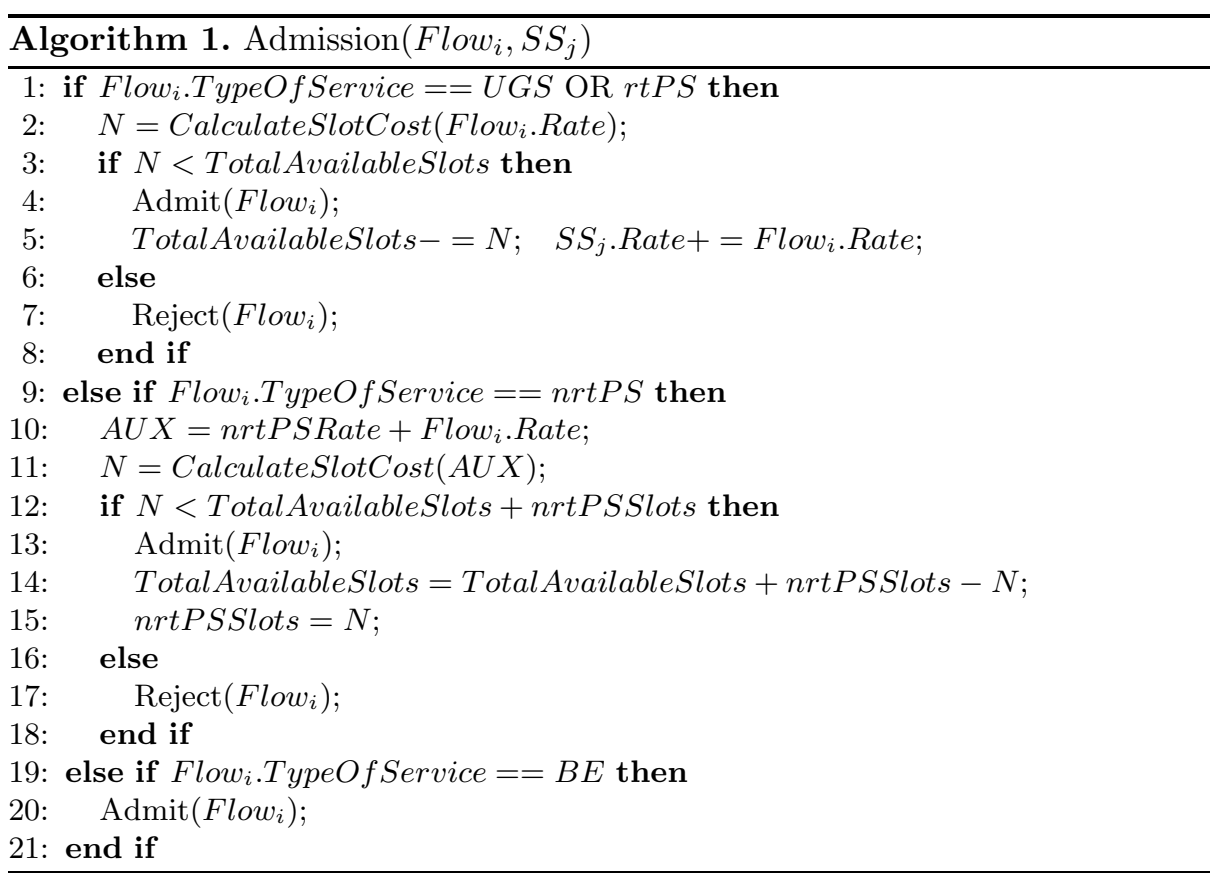

- TotalAvailableSlots is the number of unreserved uplink allocation slots. The variable is initialized to a percentage of the total number of uplink slots in a frame, the rest being reserved to prevent Best Effort flows from starving.

- $S S_{i}$. Rate represents the contract of a given $S S_{i}$, it should be initialized to 0 , it will be the sum of the mean rates of time sensitive flows of $S S_{i}$.

- nrtPSRate represents the total rate of all the admitted nrtPS connections, it should be initialized to 0 .

- nrtPSSlots are the total number of allocation slots reserved by all the nrtPS connections, it should be initialized to 0 .

Function CalculateSlotCost $(R)$ is used to calculate the number of slots of an uplink frame that must be reserved to serve a generated rate of $R$. This value can be calculated as:

$$
\operatorname{SlotCost}(R)=\frac{R * F S}{\text { StationTxRate } * \text { SlotSize }}
$$

where FS is the size of a WiMAX Frame, StationTxRate is a station's transmission rate and SlotSize is the size of the slot as earlier defined.

\subsection{Aggregating the Requests}

The complex procedure that WiMAX describes for the bandwidth request mechanisms can find its explanation in a context with few connections per SS. Each connection will have to specifically request its needs and require them to be 
answered (when it is using a high priority type of service). In such a context, the SS will not have enough margin to be flexible. However in a context where each SS is serving a high number of various connections, we think it is possible to simplify the request mechanism giving the SS the flexibility to manage an across-connection aggregated request and handle the scheduling of the granted airtime without any loss in the quality of service given to the time sensitive flows. In our proposal, the complex request mechanism presented earlier (unicast polls, multicast polls, PM bits, piggybacking) will be replaced by a single per SS aggregated request, expressing the needs of the SS, and sent in a contention less fashion (as a specific UGS connection for example). The request is sent each frame, at the end of the uplink period allocated to the SS, so that the request contains an up-to-date view of the queuing situation within the SS. The aggregated request represents a photography of the situation of the queues of the SS. It contains two fields giving the BS the needed information for the uplink scheduling. The first field called the Contracted Bytes will contain the number of bytes requested by the SS that are within the admission control contract. The second field, called the Additional Bytes will contain the requested bytes that fall beyond the contract.

Building the request. We define here the exact content of each field of the aggregated request. As defined earlier, $S S_{i}$. Rate is the sum of the mean rates of all time sensitive flows (using UGS and rtPS services). We define $W(\tau, t)$ as being the service received by the time sensitive flows during the current backlogged period (which began at $\tau, t$ being the current time). We also define $t r_{k}$ as the time of transmission of the SS's aggregate request in the current frame (which will usually be at the end of the uplink transmission time allocated to the SS as specified by the UL-MAP). Let $Q_{U G S}$ be the amount of UGS bytes enqueued within the SS at the moment the request packet is being built, the same applies to $Q_{r t P S}, Q_{n r t P S}$ and $Q_{B E} \cdot \varepsilon_{U G S}$ is the slip amount for the UGS queue: it is the amount of enqueued bytes that do not go within the contract: $\varepsilon_{U G S}=$ $\max \left(Q_{U G S}-R_{U G S}, 0\right), R_{U G S}$ being the total amount of UGS bytes falling within the contracted request. The same applies to $\varepsilon_{r t P S}$.

The aggregated request's fields are built as follows: the CB field will contain the amount of UGS and rtPS bytes enqueued within the SS that respect the SS's contract in addition to a possible slip of the UGS queue (a slip in the UGS queues is incidental); the $\mathrm{AB}$ field will contain the amounts of bytes enqueued within the SS that did not make it to the CB field (i.e. nrtPS and BE bytes in addition to rtPS bytes that exceed the contract due to the possible variation in an rtPS flow). Note that the sum of $\mathrm{CB}$ and $\mathrm{AB}$ at the time of build of the request is equal to the total number of bytes enqueued within the SS. Figure 1 shows a view of what the CB field represents.

$$
\begin{aligned}
C B & =\left(S S_{i} \cdot \text { Rate }\left(t_{k}-\tau\right)-W\left(\tau, t r_{k}\right)\right)+\varepsilon_{U G S} \\
\text { and } A B & =\varepsilon_{r t P S}+Q_{n r t P S}+Q_{B E}
\end{aligned}
$$




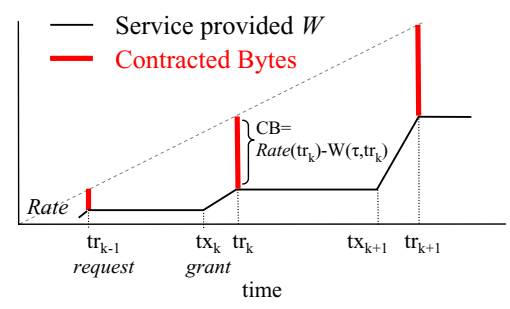

Fig. 1. The Contracted Bytes field

\subsection{Granting the Requests}

As it has been said earlier, the requests that fall under the terms of the contract of an SS should be granted for the frame that follows the one where the request was received. The possibility of this is insured by the admission control algorithm. The BS's uplink scheduling will first give each SS the number of slots necessary to transmit the Bytes indicated in the CB field of the aggregated request. The remaining slots are then to be distributed among the SSs. If there is enough slots to grant all of the additional requests, then the slots are to be distributed according to the requests, if not, the slots are distributed proportionally to the AB field of each SS's aggregated request. Algorithm 2 describes the grant procedure. We call AllocatedSlots $(i)$ the slots allocated to SS $i$, the array is initialized to $0 . C B_{i}$ and $A B_{i}$ are the translation in terms of slots of the values of the $\mathrm{CB}$ and $\mathrm{AB}$ fields of the aggregated request of SS $i . N$ is the number of SSs in the network. AvailableSlots is a variable representing the number of unallocated slots and will be initialized to the total number of slots in the uplink frame.

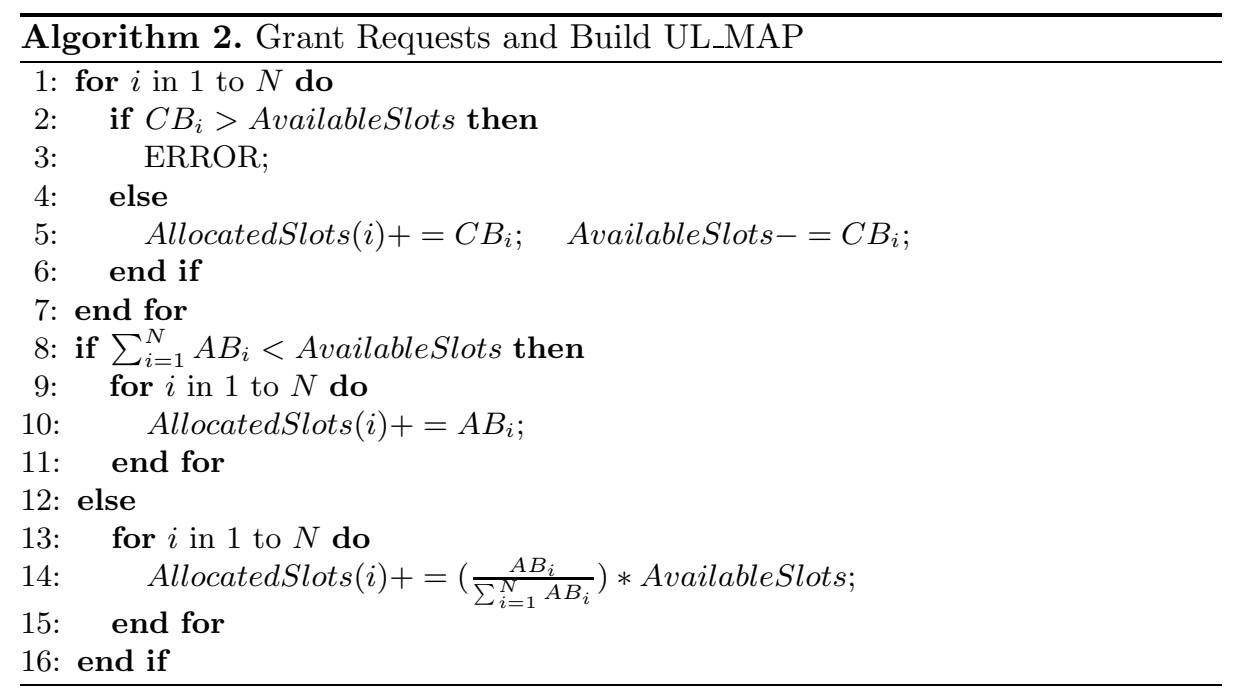




\subsection{Scheduling Algorithms}

Scheduling algorithms can be implemented at different stages of the framework, however only one of those is crucial for the correct functioning of the system which is the SS based algorithm distributing the granted slots to its different connections. This scheduling algorithm will work as follows: it will first give to each time sensitive connection the amount of slots necessary to transmit the contracted transmissions they requested. The remaining slots will then be distributed, in a round robin fashion, first to the UGS and rtPS flows needing additional airtime, then to the nrtPS connections and finally to the BE connections. This will allow us to serve as a high priority, the delay sensitive flows. nrtPS flows will be correctly served, on the long term, due to the slot reservation made by the admission control algorithm. BE flows will avoid starvation due to the percentage of bandwidth which is initially reserved. We think that once this scheduling decided, other scheduling algorithms (like scheduling the connections of one SS in the total interval allocated to the SS) will add complexity with little effects on the delays. In fact, since each SS will be granted a part of the uplink subframe (which in our case will never go beyond $2 \mathrm{~ms}$ ), scheduling the SS's connections inside this small range will have little effect on the delays.

\subsection{Characteristics of the Framework}

The service with respect to time sensitive flows conforms to an LR server model. We have proven that the designed system will act as an $\operatorname{LR}(S S$.Rate, $2 F S+$ $\left.M a x_{-} U L \_S i z e\right)$ defined in [9] giving the framework interesting properties on the rate and bounds on the delays. Due to lack of space, the proof will not be detailed herein. In our case, time sensitive flows (UGS and rtPS) will be served following an LR model which will thus give them a guaranteed rate and a higher bound on the mean access delays. We can intuitively see in figure 1 what a worst case scenario may be: a worst case being a peak arrival within contract right after the request has been sent: in a no anticipation policy a service conforming to the contracted rate starts no later than $3 F_{-} S$ (which is a higher bound for $\left.2 F S+M a x \_U L \_S i z e\right)$.

Increased determinism. In our proposal the single aggregated request of the SS is sent in a contention-less fashion, unlike standard WiMAX architecture where some bandwidth requests can be sent in contention zones. This property of the new design increases the determinism of the WiMAX bandwidth management.

Flexibility and simplicity. The aggregation of the requests and of the grants will allow a better flexibility of the bandwidth management. It will also render the whole request mechanisms simpler. The SS having a better knowledge of the state of its queues than the BS, this will allow it to adapt the request to its needs and to organize the scheduling accordingly. 
Scalability and efficiency. Our proposal will allow an efficient usage of the network resources: the BS distributing the resources will always have an updated view of the state of the SSs. Since the request and scheduling mechanisms are simple, this will allow the BS to manage a large number of connections simultaneously.

\section{Analysis}

We analyzed our proposal by mean of simulation, using the ns- 2 simulator [10]. We used the module for WiMAX proposed by Juliana Freitag Borin from the Universidade Estadual de Campinas [11. We implemented our proposal and tested it towards a standard WiMAX implementation using a scheduler presented by Freitag et. al. [5]. Freitag et. al. adopt a WiMAX classical request procedure and a per connection management of the bandwidth. Freitag et. al.'s scheduler is designed to respect throughput requirements for the time sensitive flows. The goal of the analysis is to confirm the different properties of our framework : the possibility to have a large number of simultaneously active connections, having these connections served correctly and achieving the guarantees on the rate and on the access delays of the time sensitive flows. We detail in the following paragraphs the simulation scenarios along with the results and an analysis of the results.

\subsection{Simulation Scenarios and Results}

Different scenarios were tested with variation on the number of SSs, the number of flows per SS and the rate of the different flows. The results of those different scenarios drive to the same conclusions. We chose to present one set of parameters varying only the size of the WiMAX frame. This is to show the effect of the frame size on the access delay. Time Division Duplexing is used to duplex the DL and UL subframes. The physical rate is of $40 \mathrm{Mbps}$. The network is made of a BS and $10 \mathrm{SSs}$ with the same flow profile. Within each SS, 10 flows are activated at the beginning of simulation: 3 flows using UGS (24.6 kbps CBR), 3 flows using rtPS (64 kbps ON-OFF), 3 flows using nrtPS (64 kbps CBR) and 1 flow using BE (1 Mbps CBR). Simulations are 20 seconds long. Figures 2 thru 7 present different results with frame size varying from 2 to $10 \mathrm{~ms}$ (we varried FS in our simulations from 2 to $20 \mathrm{~ms}$ ). The results are one of the following:

- a cumulative distribution function (CDF) of the access delays of time sensitive flows (UGS and rtPS),

- a CDF of the access delays of nrtPS and BE flows,

- throughput of either nrtPS or BE flows.

We chose not to show graphs of the throughput of UGS and rtPS flows as they bear little information: UGS and rtPS flows achieve their generation rate. 


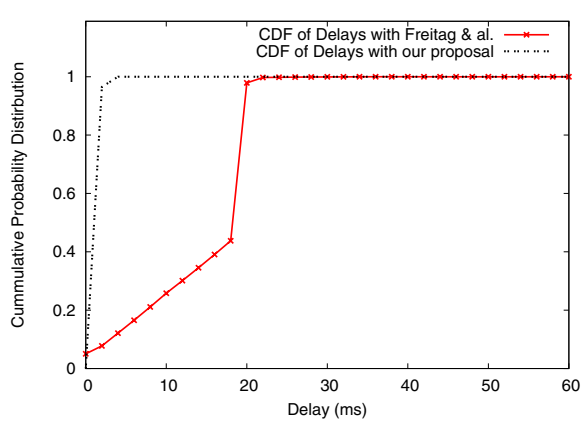

Fig. 2. Delays of UGS and $\operatorname{rtPS} F S=2 \mathrm{~ms}$

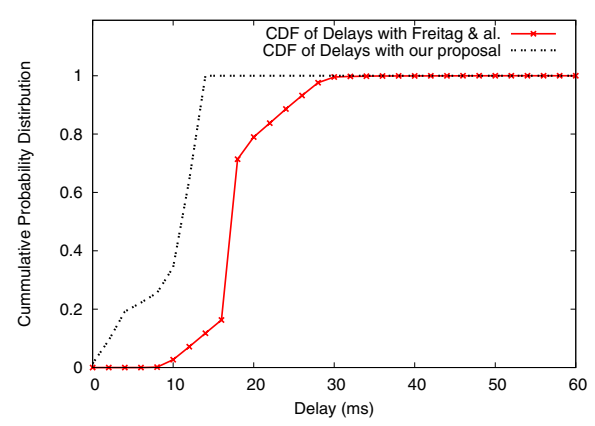

Fig. 3. Delays of UGS and rtPS FS $=10 \mathrm{~ms}$

\subsection{Interpreting the Results}

The Access Delays. Our proposal achieves better access delays in all cases, for all kinds of flows (figures 2 to 5 allow us to compare the CDF of access delays of different flows in different scenarios using our framework or Freitag et. al.'s), we can clearly see that in all cases, our framework achieve a better overall access delay for all kinds of flows. The huge difference in delays of NRTPS and BE is due to the way WiMAX functions: WiMAX will seldom allow these kinds of flow to express their needs which is not the case in our scheme thanks to the aggregated bandwidth request. Moreover, the guarantee on the access delay of time sensitive flows can be clearly seen. In figures 2 and 3, the time sensitive flows achieve access delays that are at most equal to the double of the frame size. This is due to our scheduling policy, forcing a time sensitive request to be granted at the frame following the one bearing the request.

The Throughput. Our proposal achieves a better overall throughput going from $130 \%$ to $267 \%$ better if compared to the results of Freitag et. al. We see that our proposal reduces the waste in uplink resource since the BS is permanently

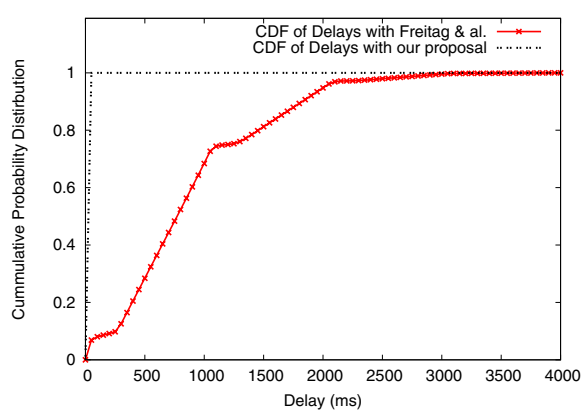

Fig. 4. Delays of NRTPS FS $=10 \mathrm{~ms}$

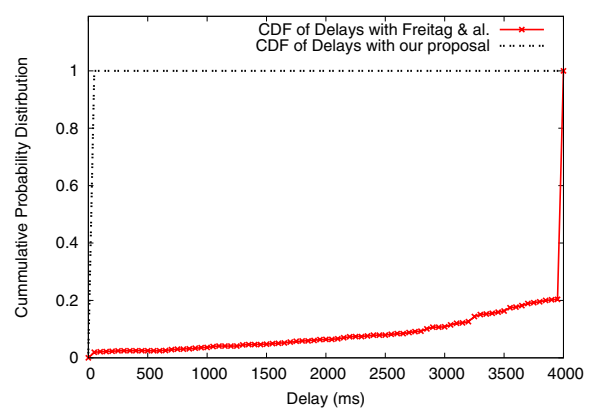

Fig. 5. Delays of BE FS=10ms 


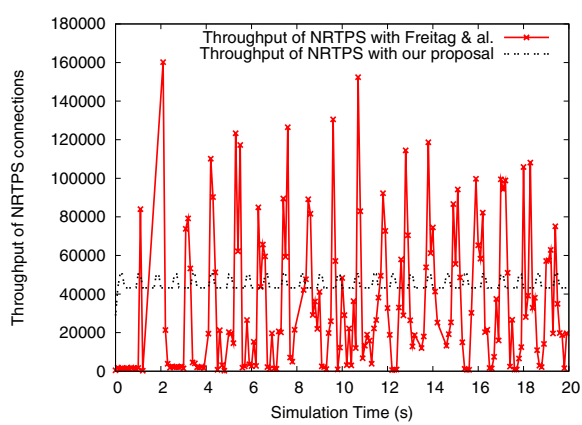

Fig. 6. Throughput of NRTPS FS=10ms

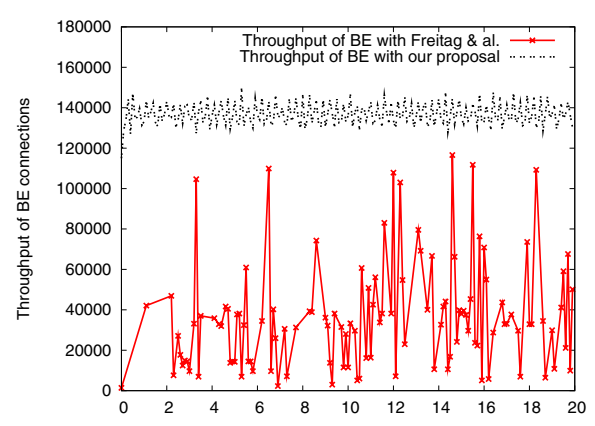

Fig. 7. Throughput of $\mathrm{BE} F \mathrm{~F}=10 \mathrm{~ms}$

updated with the situation of the queues within the SSs, giving it more flexibility to manage the bandwidth as needed. Classical WiMAX request mechanisms will give the BS scarce information about the situation of nrtPS and BE queues, making it waste precious resources when it lacks this information especially with a larger frame size. Another interesting aspect of our proposal is the stability of the service given to these flows as can be seen in figures [6] and 7. For example, in figure 6] the service given to nrtPS flows by our framework is stable around 40 KBps whereas the service offered by the Freitag et. al. is more varying. Allowing these flows to express their needs in the SS's aggregated request is the main cause of this behavior.

Conclusions. Our proposal proved to perform better than the standard WiMAX along with the scheduler at several levels: time sensitive flows were given better delays. The framework insured better delays for nrtPS and BE flows as well with a better throughput and much more stable service, even though a high number of connections are simultaneously active in the network.

\section{Conclusion}

We propose in this paper a bandwidth management framework for IEEE 802.16 uplink access combining a modification of the WiMAX bandwidth request mechanisms, an admission control algorithm and an uplink scheduling algorithm. This paper details the different algorithms used in our framework. The framework was analyzed using simulation and compared to standard WiMAX procedure and a scheduling algorithm proposed in [5]. Our framework allows giving guarantees on the rate and the access delays of time sensitive flows. Our framework proved to achieve an overall better usage of the network resources and achieved better access delays for the different types of service while adopting a simple procedure at the BS level allowing it to manage a large number of connections. This work will further be developed and integrated in a heterogeneous WiFi-WiMAX network in order to give a global QoS solution for heterogeneous wireless networks. 


\section{References}

1. 802.16: IEEE Standard for Local and Metropolitan area Networks Part16: Air Interface for fixed broadband Wireless Access Systems (2004)

2. Forum, W.: Business Case Models for Fixed Broadband Wireless Access based on WiMAX Technology and the 802.16 Standard (2004)

3. Liu, Q., Wang, X., Giannakis, G.: A cross-layer scheduling algorithm with qos support in wireless networks. IEEE Transactions on Vehicular Technology 55(3), 839-847 (2006)

4. Niyato, D., Hossain, E.: Queue-aware uplink bandwidth allocation and rate control for polling service in ieee 802.16 broadband wireless networks. IEEE Transactions on Mobile Computing 5(6), 668-679 (2006)

5. Freitag, J., da Fonseca, N.: Uplink scheduling with quality of service in ieee 802.16 networks. In: Global Telecommunications Conference, 2007. GLOBECOM 2007, pp. 2503-2508. IEEE, Los Alamitos (2007)

6. Delicado, J., Orozco-Barbosa, L., Delicado, F., Cuenca, P.: A qos-aware protocol architecture for wimax. In: Canadian Conference on Electrical and Computer Engineering, 2006. CCECE 2006, May 2006, pp. 1779-1782 (2006)

7. El Masri, M., Abdellatif, S., Juanole, G.: Proposal of a novel bandwidth management framework for ieee 802.16 based on aggregation. In: New Technologies, Mobility and Security, 2008. NTMS 2008, pp. 1-5 (2008)

8. Oh, S.M., Kim, J.H.: The analysis of the optimal contention period for broadband wireless access network. In: Third IEEE International Conference on Pervasive Computing and Communications Workshops, 2005. PerCom 2005 Workshops, pp. 215-219 (2005)

9. Stiliadis, D., Varma, A.: Latency-rate servers: a general model for analysis of traffic scheduling algorithms. IEEE/ACM Trans. Netw. 6(5), 611-624 (1998)

10. ns 2: The network simulator ns-2, http://www.isi.edu/nsnam/ns/

11. Freitag, J., da Fonseca, N.: Simulator for wimax networks. Simulation Modelling Practice and Theory 16(7), 817-833 (2008) 